\title{
Minicomputer labs that talk to maxicomputers
}

\author{
EDWARD GRUPSMITH and STEVEN FERRES \\ New York University, New York, New York 10003
}

Limitations of the minicomputer system for performing large-scale data analysis are discussed.

Computer systems are developed for different purposes. The purposes behind the development of one system may create certain advantages for that system over one developed for other reasons. On the other hand, certain disadvantages may result as well. For instance, a system designed for research may not be as easily used for data analysis as one that has been specifically designed for that purpose and vice versa. Minicomputer and maxicomputer systems are each generally better suited to perform different tasks than the other. Hence, they should be used for their respective purposes when there exist such internal constraints as those exemplified by hardware or software differences, or external constraints such as time or money.

The psychologist who buys a minicomputer system in order to do research may lose sight of the fact that his system may not be the optimal one on which to perform data analyses. The tendency to use the minicomputer system for both purposes may result from the desire to have a self-sufficient laboratory. However, self-sufficiency in the normal minicomputer environment may have disadvantages as well as advantages. On the plus side lies the significant feature of being removed from the "outer world" administrative politics that may slow research. Second, self-sufficiency implies no need to use external resources, so there is no reason to transfer data from one location to another in order to analyze them. One disadvantage involves the fact that psychology departments generally have fewer trained programmers and staff advisors available to serve the needs of the users than university centers do. Large computer centers pool resources from many departments and thus reduce the effective or relative cost per user. Second, the average minicomputer laboratory has fewer facilities than the large computer environment, in terms of both hardware and software. The small laboratory is likely to have much less money available to boost the potential of its hardware and software. These drawbacks can result in drastic mistakes in time and money in the attempt to compensate or overcome the deficiencies.

This work was supported in part by USPH Grant MH 16496 to D. Aaronson. We thank D. Aaronson for helpful comments on earlier drafts.

\section{LIMITATIONS OF THE MINICOMPUTER FOR DATA ANALYSIS}

Let us consider in more detail the limitations of the minicomputer system for performing large-scale data analysis.

Most psychologists buy minicomputers for the purpose of doing experimental research. These computers are generally not optimally configured for performing large-scale data analyses. However, many researchers make the mistake of using these machines for data analysis in the attempt to become selfsufficient. Problems arise because of the limitations of available hardware and software resources, as well as their interaction.

Minicomputer systems have less standard statistical software than do large systems. What software does exist has less depth and versatility and can be applied to fewer problems. Large computer systems have readily available packages such as BMD, BMDP, SPSS, DATATEXT, and SAS, which are highly versatile and useful in performing statistical analyses. These packages have been designed by professional programmers in order to maximize efficiency and minimize cost. In addition, they have been extensively tested and debugged. If no standard statistical packages are suitable to the user's purpose, there exist subroutine libraries of mathematical programs that enable the user to quickly and easily create his own analysis programs. The researcher who is without these analysis capabilities must create his own programs from scratch. This can be extremely time consuming, taking away from valuable research time and money meant for other purposes. There is also the risk that a program will be implemented that will create the illusion of performing its function while not really doing so.

Minicomputer systems are also more limited in their hardware resources than are large systems. One obvious limitation is size. While the small systems are entirely adequate for interactive experimental situations, they are insufficient for complicated data analyses. Also, the average laboratory is likely to have a smaller size than its maximum could be. In a recent survey of DEC-equipped psychology computer laboratories (Aaronson, 1979), of 25 laboratories that responded 19 had systems that were configured with $32 \mathrm{~K}$ or less 
core. Another hardware limitation in the small systems is the availability of high-speed printed output. In the same survey, there were only six respondents who had printers that ran faster than 30 characters/sec. Minicomputers also have slower cycle speeds than do large computers, resulting in longer computation speeds, an important feature in data analyses. Finally, small systems have smaller word sizes, which leads to less accuracy in mathematical computations.

The attempt to write data analysis programs for a small system can cause the hardware and software limitations to interact with each other, resulting in further problems. The minicomputer user who tries to write his own software program may find that it does not all fit into core at once. This leads to breaking the program into several segments, each of which must be loaded separately, creating a more complex program structure and adding to program running time. Also, the data needed for analysis by the program may not fit into core simultaneously with the program. This necessitates the extensive use of external storage devices. For a laboratory without timesharing capabilities, the use of the computer for data analysis precludes experimental use. For larger timesharing minicomputers, the data analysis may take time away from simultaneously running experiments. This could result in more variable reaction time data for systems reasons, or simply because the subject does not experience a smoothly and rapidly running session (Aaronson \& Brauth, 1972).

To summarize, minicomputer systems are often inadequate for performing large-scale data analyses. Hardware and software limitations interact to create programming and execution problems that may affect both data analysis and on-going experimental research. One solution is to use large computers for data analysis whenever possible.

\section{METHODS OF LINKING LARGE AND SMALL COMPUTER SYSTEMS}

Given that a researcher has decided to use a large computer, how does he go about setting up communications between it and his laboratory? There are at least four methods, all of which have advantages and disadvantages.

One medium that is compatible with the large computer is the card punch. Its advantage is its universality. However, the cost of a card punch for a minicomputer is high, ranging from $\$ 4,000$ to $\$ 8,000$, and its output is very slow and bulky. Finally, unless a card reader is also purchased, the output is virtually unusable on the minicomputer system on which it was punched.

A second compatible medium is magnetic tape, which is compatible with virtually all large computers. In addition, it allows compact storage of large amounts of information and adds to the on-line and off-line mass storage potentials of the minicomputer system. Unfortunately, magnetic tape drives and controllers are extremely costly. A small drive and controller can be purchased for about $\$ 6,000$, but a full-scale highdensity tape drive costs about $\$ 20,000$. Another possible disadvantage is that the main computer center may be located at some distance from the minicomputer, so there may be some difficulty in communications. Transporting the tapes between the two locations may cause time and convenience problems.

Telecommunications provide a third method of attaining capability between large and small machines; costs may be high or low. In our laboratory, a low-cost telecommunications link has been implemented. The only requirement for this type of link is an acoustic coupler, which can be attached to an already existing serial interface. A typical acoustic coupler can be purchased for less than $\$ 300$. Another advantage of this type of link is that it can be used at any time without leaving the laboratory, and we can maintain direct interaction between the large and small computers. The disadvantages of this set-up include the slow transmission rates available, which may range from 30 to 120 characters/sec. Also, a program must be written to enable the minicomputer to act as a terminal to the large main frame. However, this may not be a major problem, since there already exist several programs that have solved this problem (Grupsmith, 1978; Vaughan, 1978). Finally, a hidden cost in this type of arrangement may be the telephone bill, if public lines are used. This factor may sway the researcher's decision to use tape, which has a high initial cost but low future costs. Aaronson's (1979) survey found that 7 of 25 laboratories have big magnetic tape systems; 8 had phone modems.

Higher cost telecommunications can also be used to link computers. Their advantages include the availability of previously developed hardware and software. For example, hardware and software are available for PDP-1 1s that enable the computers to work as remote job entry stations to larger machines. If the computer center permits dial-up RJEs and the user has large amounts of data to transmit, this may be a viable alternative to a low-cost telecommunication system. A further advantage of the high-cost telecommunications is that they are much faster than their low-cost counterparts. Transmission rates of 200 to 240 characters/sec are commonly available, and if a leased telephone line is installed, speeds up to 960 characters/sec can be obtained. The former type of system costs from $\$ 550$ to $\$ 1,500$; the latter type costs at least $\$ 3,600$ without a private line. A private line may cost up to $\$ 1,000$ for installation and demand a substantial monthly bill, which varies with distance from the large computer. If the user is within 5 miles of his computer center, he can utilize limited-distance modems, which combine the advantages of high speed and low cost. A limited distance modem can be purchased for as little as 
$\$ 550$. A final advantage of the higher cost telecommunications is that they can provide better errorchecking capabilities.

In conclusion, using minicomputers designed for experimental research purposes for performing large-scale data analyses may cause many problems. These problems are related to the hardware and software capabilities and limitations of the minicomputers, as well as to factors outside of the machine that may come into play. We feel that data analysis belongs on a large main frame, where it can be done more efficiently, Minicomputers should be used for their originally intended purpose, namely, research. This saves the researcher both time and money. The solution that we have chosen is a low-cost telecommunications link between the large and small machines.

\section{REFERENCES}

AARonson, D. DEC vs. DEC-compatible hardware. Behavior Research Methods \& Instrumentation, 1979,11, 159-162.

Aaronson, D., \& BRAuTH, S. Simple guidelines for developing a computer based laboratory. Behavior Research Methods \& Instrumentation, 1972, 4, 257-264.

GRUPSMITH, E. Linking large and small computers. Behavior Research Methods \& Instrumentation, 1978, 10, 269-272.

Vaughan, J. The mini and micro as intelligent terminals. Behavior Research Methods \& Instrumentation, 1978, 10, 264-268. 